\title{
Factores que predicen el rechazo escolar a largo plazo
}

\author{
Eila Laukkanen, $\mathrm{PhD}^{*}$ \\ Pirjo Pölkki, PhD** \\ Liisa Oranen, $M A^{\star * *}$ \\ Heimo Viinamäki, $\mathrm{PhD}^{\star \star \star *}$ \\ Johannes Lehtonen, $\mathrm{PhD}^{\star \star \star *}$ \\ * Psiquiatría de Adultos y Adolescentes, \\ Kuopio University Hospital, \\ P.O. Box 1777, FIN-70211 Kuopio \\ ** Lecturer Senior en Psicología, \\ Department of Social Sciences, Kuopio \\ University, P.O. Box 1627, FIN-70211 Kuopio \\ *** (Educ.), Vice Principal, Kallavesi \\ Comprehensive School, Blominkatu 2, \\ FIN-70820 Kuopio \\ **** Profesor, Departamento de Psiquiatría, \\ Kuopio University and Kuopio University \\ Hospital, P.O. Box 1777, FIN-70211 Kuopio \\ FINLANDIA
}

\begin{abstract}
RESUMEN - Se estudiaron los factores que podían predecir el rechazo escolar entre 217 alumnos de 13 años de edad al inicio del estudio y que alcanzaron los 16 años durante el seguimiento. El rechazo quedó determinado a través de una evaluación sociométrica que realizaron los alumnos. Al comienzo del estudio, los profesores evaluaron los problemas de comportamiento de los alumnos así como sus habilidades sociales por medio de Escalas de valoración de habilidades sociales. La imagen que tienen de sí mismos se midió mediante el Cuestionario de Autoimagen de Offer y también se valoró el éxito académico. Los factores que, en un principio,predecían el rechazo a largo plazo eran el sexo masculino (odds ratio [OR 7,2 95\% intervalo de confianza [IC 1,2 - 41,5), bajo rendimiento en Educación física (OR 6,4 95\% IC1,8 - 20,0), imagen negativa sexual de sí mismo (OR 2,3 95\% IC 1,5 - 3,6) y problemas de interiorización (OR 4,3 95\% IC 2,0 - 9,2). El conocimiento de los factores de riesgo inherentes al rechazo escolar pueden ser valiosos a la hora de planificar actividades preventivas.
\end{abstract}




\section{Introducción}

Ser rechazado por aquellos que te rodean supone un riesgo para el desarrollo psíquico durante la adolescencia. La buena relación entre compañeros suele favorecer muchas etapas en el desarrollo tales como la separación de los padres (Forehand et al. 1991, Resnick et al. 1997), el descubrimiento de la identidad sexual y profesional, la formación de opiniones sobre la vida y la propia preparación para el establecimiento de nuevas relaciones que implican emociones (Blos 1962, O'Koon 1997, Youniss \& Haynie 1992). Los contactos sociales también contribuyen a que los adolescentes puedan hacer frente al estrés diario y a que dominen habilidades que le servirán para desenvolverse en diferentes situaciones sociales (Shulman 1993, Laukkamen et al. 1998). Por otro lado, la relación con los amigos puede hacer que los adolescentes tiendan hacia actitudes antisociales o al abuso de estupefacientes (Downs \& Rose 1991, Kandel \& Davies 1996). La importancia subjetiva que tiene la relación entre los amigos queda reflejada en artículos sobre la juventud que, en su mayor parte, tratan sobre las relaciones con los amigos y con los padres (Levine 1981).

La pubertad también puede afectar a las relaciones sociales de los adolescentes. Algunos investigadores han elaborado hipótesis que sugieren que el cambio de la pubertad es más estresante cuando sitúa al joven adolescente en un estatus diferente al de sus amigos e incluso puede llegar a afectarle psicológica y socialmente (Petersen 1988).

La sociometría es una forma de valorar el estatus social de los alumnos en una clase. La clasificación sociométrica más común que se ha utilizado es 1. popular 2. descuidado 3 . polémico (tanto positiva como negativamente) 4. rechazado (Coie, Dodge \& Kupersmidt 1990). Hasta ahora, los estudios sociométri- cos han sido llevados a cabo en la mayoría de las ocasiones con niños y, en raras ocasiones, entre adolescentes. Un estudio realizado por Francio, Davis y Vasquez-Suson (1994) (los alumnos estudiados cursaban el primer ciclo de Educación Secundaria), mostró que el estatus en clase era más estable en adolescentes que en chicos con edades menores.

Es importante destacar que los alumnos más populares tienden a obtener mejores calificaciones (Wentzel \& Caldwell 1997), a menudo buscan mejorar su educación, socialmente son más activos y gozan de una autoestima mayor que la de aquellos que son ignorados o discriminados por el grupo. Por otro lado, el hecho de ser el objeto de burlas por parte del resto de sus compañeros de clase, ha sido relacionado con un mal rendimiento académico y una autoestima baja (Boivin, Hymel \& Bukowski 1995). Los estudios sobre intimidaciones y burlas en la escuela muestran cómo los alumnos rechazados son además, los que más las sufren (Salmivalli et al. 1996). Un estudio sobre población adolescente finlandesa de entre 14 y 16 años de edad, mostró que la prevalencia de depresión así como de ideaciones suicidas graves aumenta entre aquellos que sufrieron burlas y entre los que las realizaban (Kaltiala-Heino et al. 1999).

Del mismo modo, diferentes estudios de seguimiento han observado que el grupo con mayor riesgo, en lo que a la salud mental y al éxito profesional se refiere, está formado por alumnos que habían sido rechazados (Newcomb, Bukowski \& Pattee 1993). Roff y Wirt (1984) descubrieron que el riesgo de terminar convirtiéndose en un paciente psiquiátrico, incluso durante la adolescencia, es 2 ó 3 veces mayor para los que han sido rechazados que para el resto. Kupersmidt y Cole (1990) observaron que los niños que sufrían este rechazo a la edad de 11 años tenían más problemas al llegar a los 18 . Por otro lado, Giordano y sus colaboradores 
(1998) han mostrado en su estudio de seguimiento de 10 años que la calidad en la relación con los padres era más importante que la que existía con los amigos. Sin embargo, el estatus sociométrico estaba unido al número de amigos y al apoyo social durante la edad adulta. Por otro lado como los adolescentes pasan gran parte de su tiempo en la escuela, el estrés ante la posibilidad de ser rechazados en clase es ciertamente muy alto.

Por ello investigamos si existía algún factor que, a la edad de 13 años, pudiera predecir el rechazo en los cursos $7^{\circ}$ y $8^{\circ}$ (los así llamados rechazos a largo plazo) durante los años más significativos (de 13 a 16) del desarrollo del adolescente. Estábamos especialmente interesados en obtener respuesta a las siguientes preguntas:

1. ¿Pueden las habilidades sociales y los problemas psicológicos de los alumnos llegar a predecir el rechazo a largo plazo?

2. ¿Son los éxitos escolares diferentes para aquellos que han sido rechazados durante mucho tiempo que para otros alumnos en los que ello no ha sucedido?

3. ¿Es el entorno psicológico de los alumnos que han sido rechazados durante mucho tiempo, diferente al de aquellos que no lo han sido?

4. ¿Existen diferencias en cuanto a la duración de la pubertad entre los alumnos que han sufrido rechazo durante mucho tiempo y los demás?

\section{Métodos}

\section{Muestra}

En nuestro análisis inicial nos dedicamos a estudiar a todos los alumnos de $7^{\circ}$ curso de dos centros de Educación Secundaria en
Finlandia (244 adolescentes de 13 años \pm 3,9 meses). El número de alumnos por clase variaba entre 17 y 25 , siendo 20 el término medio. Estos alumnos permanecían juntos desde $7^{\circ}$ curso hasta $9^{\circ}$. Aquellos que fueron trasladados a clases especiales se excluyeron de nuestro estudio. Conseguimos el consentimiento de los padres y de los adolescentes por adelantado, así 235 adolescentes participaron en la evaluación inicial (en 1995). Tres años después, se llevó a cabo un estudio de seguimiento (en 1998). Durante este seguimiento, el número de alumnos era de 217 y el $92 \%$ había participado en el estudio inicial. Sólo 10 alumnos habían cambiado de escuela.

En el estudio inicial, el $72 \%$ de los alumnos pertenecían a familias con un padre y una madre, el $19 \%$ a familias con sólo uno de los dos padres y el $9 \%$ a familias no nucleares. La clasificación se realizaba según la profesión del padre (Clasificación de grupos socioeconómicos, 1989), del siguiente modo: obreros $37 \%$, empleados de cuello blanco de nivel bajo, $25 \%$, empleados de cuello blanco de nivel alto $25 \%$, autónomos $7 \%$ y desempleados de larga duración $6 \%$.

\section{Cuestionario Sociométrico}

Con el fin de detectar a los alumnos que sufrían rechazo, utilizamos un cuestionario sociométrico (Stensaasen 1965) que ya había sido utilizado anteriormente por Aho (1979) en Finlandia. Pedimos a los alumnos que clasificaran a sus compañeros partiendo de la siguiente base: 1 . Haz una lista con los alumnos de tu clase que son respetados y estimados por otros alumnos (líderes positivos). 2. Haz una lista de los alumnos de tu clase que caen bien a otros alumnos (populares). 3. Haz una lista de los alumnos de tu clase con quienes otros compañeros no 
quieren estar (rechazados). 4. Haz una lista de los alumnos de tu clase que sufren burlas y ofensas por parte de sus compañeros (intimidados).

En base a las respuestas obtenidas se formaron dos grupos, los que eran rechazados $y$ los que no. Aquellos a quienes al menos dos de sus compañeros calificaron como rechazados o como objeto de burla (preguntas 3 y 4) y a los que nadie consideró como populares o líderes positivos (preguntas 1 y 2), fueron clasificados como rechazados. Se repitieron los mismos cuestionarios sobre el estatus sociométrico tres años después y las respuestas fueron clasificadas del mismo modo.

\section{Cuestionario de Autoimagen de Offer (OSIQ)}

El Cuestionario de Autoimagen de Offer (OSIQ) es un test autodescriptivo de personalidad cuyo objetivo es valorar los sentimientos de los adolescentes respecto a su propio mundo psicológico en 12 áreas de contenido. El Cuestionario de Autoimagen de Offer (OSIQ) consiste en 130 items de seis clases, formando 12 escalas (S1-S12) (Offer et al. 1992). Se ha demostrado que la validez del test es bastante aceptable para el desarrollo normal (Lukkanen, Halonen \& Viinamäki 1999a, Laukkanen et al. 1999b). La consistencia interna de la suma de puntuaciones fue examinada por Cronbach's $\alpha$. Todos los alumnos completaron los cuestionarios a la vez. En este estudio, se utilizó la suma de todas las escalas (130 items). Las puntuaciones también se calcularon de forma independiente para las siguientes escalas: S1: control de impulsos (9 items, $\alpha$ $=.65), \mathrm{S} 2$ Tono emocional (10 items, $\alpha=$ .74), S3 Imagen corporal (9 items, $\alpha=.72$ ), S4: relaciones sociales (9 items, $\alpha=.69$ ) S6: sexualidad (10 items, $\alpha=$.71) S7: rela- ciones familiares (19 items $\alpha=.80$ ) y S10: (14 items, $\alpha=.69$ ) salud emocional. Una alta puntuación total refleja una percepción individual negativa de sí mismo o de sí misma.

\section{Escala de valoración de las habilidades sociales}

Los profesores completaron una Escala de Habilidades Sociales, en la que reflejaban los comportamientos conflictivos (hiperactividad, interiorización y exteriorización de los problemas) y las habilidades sociales de los alumnos (Gresham \& Elliot 1990). La escala muestra diferentes niveles, concretamente "con que frecuencia surge determinado comportamiento social ( $1=$ nunca, $2=$ algunas veces, $3=$ con frecuencia y con mucha frecuencia)". Se analizó la suma de las puntuaciones relacionadas con estas tres escalas. Las fiabilidad de las escalas era buena: hiperactividad, $\alpha=.92$ (6 items, por ejemplo actos impulsivos, problemas de concentración), problemas de exteriorización, $\alpha=.90$ (6 items, por ejemplo, peleas con otros, sustos o intimidaciones a otros), problemas de interiorización, $\alpha=.86$ (6 items, por ejemplo, parece solitario, muestra ansiedad y depresión). Se valoraron las habilidades sociales utilizando una escala de 6 items. Estos items eran: cooperación con los profesores, cooperación con sus compañeros de clase, empatía, asertividad, autocontrol y responsabilidad $(1=$ mal, $2=$ bastante mal, 3 = bien, 4 = excelente). Se analizó la suma de las puntuaciones de estos items $(\alpha=.87)$.

\section{Éxitos académicos}

Los éxitos académicos de los alumnos se evaluaron utilizando los boletines escolares 
de fin de curso en $7^{\circ}$ (inicio del estudio) (intervalo de 4 a 10). Los datos recogidos se utilizaron como media para valorar los cursos y asignaturas de la lengua madre (finlandés), segunda lengua oficial (sueco), matemáticas y educación física. La asignatura de educación física en una escuela finlandesa incluye la educación para la salud, los deportes y los juegos tanto en grupos como de forma individual.

\section{Periodo de la pubertad}

La información sobre el comienzo de la pubertad para cada alumno se obtuvo gracias a los datos ofrecidos por la enfermera del centro: Comienzo de la menstruación/ eyaculación así como variación de la curva de crecimiento.

Comparamos a los alumnos que padecían rechazo de forma crónica $(\mathrm{N}=16)$ con aquellos que no lo padecían $(\mathrm{N}=168)$ de forma aleatoria (tabla I). El estatus sociométrico (rechazado $v s$ otro) era bastante constante (kappa $=0,40)$. Entre las variables que se encontraban en el inicio del estudio, buscamos factores que pudieran predecir el rechazo.

Se utilizó el test "Chi-cuadrado" y el test de dos colas de Fisher para evaluar las diferencias entre las variables de cada clase así como el test "t de Student" de datos pareados para valorar las diferencias en variables continuas. Se calcularon los odds ratio (OR) con intervalos de confianza del $95 \%$ por medio de un análisis de regresión logística multivariable con el fin de evaluar la independencia de los factores que determinan un rechazo a largo plazo y para controlar aquellos factores que pueden dar lugar a confusión.

\section{Resultados}

Dieciséis alumnos (cinco niñas y once niños) fueron rechazados en su clase tanto a la edad de 13 años como a la de 16 años (rechazados de forma permanente). El sesenta y tres por ciento de aquellos que habían sido rechazados (otros estudios obtuvieron el $68 \%$, dato que no es significativo) habían alcanzado la pubertad a la edad de 13 años.

De acuerdo con la evaluación realizada por los profesores, aquellos que sufrían rechazo de forma permanente, presentaron, durante el inicio del estudio, más problemas subjetivos como por ejemplo, soledad, tristeza, depresión y ansiedad, que los alumnos que no sufrían rechazo. Los alumnos que padecían rechazo, de una forma habitual, también presentaban más problemas de hiperactividad en clase que el resto de sus compañeros. Sin embargo, no se detectaron diferencias significativas entre los grupos en

Tabla I

Estatus sociométrico a la edad de 13 años y 3 años después

\begin{tabular}{lccc} 
A la edad de 13 años & $\begin{array}{c}\text { A la edad de } 16 \text { años } \\
\text { No rechazados } \\
\mathrm{N}\end{array}$ & $\begin{array}{c}\text { Rechazados } \\
\mathrm{N}\end{array}$ & Total \\
\hline No rechazados & 168 & 12 & $\mathrm{~N}$ \\
Rechazados & 21 & 16 & $37(82 \%)$ \\
Total N & $189(87 \%)$ & $28(13 \%)$ & 217 \\
\hline
\end{tabular}

Kappa $r=0,40 p=0,0001$. 
cuanto a los problemas de exteriorización o en cuanto a las habilidades sociales en el inicio del estudio (tabla II).

Los alumnos que, en el inicio del estudio, habían sido rechazados obtuvieron puntuaciones más bajas en educación física que en el resto de las asignaturas, pero no había otras diferencias que se asociaran con los éxitos académicos (tabla II).

En el inicio del estudio, las puntuaciones totales del OSIQ en los alumnos que habían sido rechazados no mostraban diferencias importantes con las puntuaciones OSIQ de los alumnos que no sufrían rechazo, sin embargo, las puntuaciones eran significativamente más altas en aquellas escalas que describen las relaciones sociales (S4) y las tendencias sexuales (S6) (tabla II). El cuarenta y siete por ciento de los padres de los chicos que sufrían rechazo eran obreros (otros estudios obtuvieron un resultado del $37 \%$, pero este dato no es significativo). El treinta y siete por ciento eran empleados de cuello blanco (el 25\% para otros estudios). El setenta y cinco por ciento de aquellos que eran rechazados por el resto de sus compañeros vivían con los dos padres (otros estudios obtuvieron un $71 \%$, éste no es un dato significativo).

Los factores asociados de forma independiente con un rechazo a largo plazo fueron evaluados por medio de un análisis de regresión logística utilizando el método Enter. Aparte del sexo, en el modelo se incluyeron las variables que, en el inicio del estudio, habían determinado una diferencia estadísticamente significativa entre los alumnos que habían sufrido rechazo y los demás. Estas variables incluían la puntuación relacionada

\section{Tabla II}

Valor predictivo de las valoraciones de los profesores (Escala de Habilidades Sociales), autoimagen (Cuestionario de Autoimagen de Offer (OSIQ)) y éxitos escolares en relación con el rechazo a largo plazo en muchachos de 13 años de edad

$\begin{array}{cc}\text { Largo plazo } & \text { Grupo no rechazado } \\ \text { Grupo rechazado }(\mathrm{N}=16) & (\mathrm{N}=168) \\ \text { M (S.D.) } & \text { M (S.D.) }\end{array}$

Escala de Habilidades Sociales:

Internalización de problemas

Media

Externalización de problemas

Media

Puntuación media hiperactividad

Media Escala Habilidades

Autoimagen:

Valor total medio OSIQ

$\mathrm{S} 1$ valor medio

$\mathrm{S} 2$ valor medio

$\mathrm{S} 3$ valor medio

$\mathrm{S} 4$ valor medio

S6 valor medio

S10 valor medio

Éxitos escolares:

Todas las asignaturas (media)

Lengua materna (media)

Lengua extranjera (media)

Matemáticas (media)

Educación física (media)

$$
10,9(2,7)
$$

$7,5(2,4)$

$7,3(1,6)$

$17,3(3,1)$

$428,7(17,1)$

$21,9(59)$

$23,6(6,8)$

$21,8(7,7)$

$23,6(6,5)$

$31,4(8,7)$

$30,2(9,0$

$7,9(0,6)$

$7,8(1,1)$

$7,8(1,3)$

$7,5(1,2)$

$7,3(0,7)$
$8,1(2,1)^{* * * *}$

$8,2(3,0)$

$9,3(4,1)^{* * *}$

$17,8(3,4)$

432,1 $(26,1)$

$22,4(5,8)$

$22,3(6,5)$

$22,8(6,4)$

$19,9(5,9)^{*}$

$27,1(6,4) *$

$31,6(8,8)$

$8,0(0,8)$

$8,0(1,1)$

$7,8(1,0)$

$7,7(1,3)$

$8,1(0,9) * * *$ 
con problemas de interiorización; la puntuación relacionada con problemas de hiperactividad; la puntuación relacionada con la escala de autoimagen que describe la sexualidad (S6); la puntuación relacionada con la escala de autoimagen que describe las relaciones sociales (S4) y las calificaciones en educación física.

Las calificaciones obtenidas en educación física resultaron ser un factor independiente asociado con el rechazo a largo plazo (OR $6,495 \%$ IC 1,8 - 20,0 p = 0,004). Se observó también una disminución en las calificaciones de Educación física debido al riesgo creciente del rechazo de una forma permanente 6,4-fold. Otros factores que estaban asociados de forma independiente con el rechazo eran el sexo (varón (OR 7,2, 95\% IC 1,2 $41,5 ; \mathrm{p}=0,03)$, la imagen sexual que cada uno tiene de sí mismo (OR 2,3 95\% CI 1,5 3,$6 ; \mathrm{p}=0,03$ ) (valores calculados por el aumento de cinco puntos de S6), y la puntuación relacionada con los problemas de interiorización (OR 4,3 95\% IC 2,0 - 9,2; p $=0,056$ ) (valores calculados por la subida de cinco puntos en la puntuación).

\section{Discusión}

Como observó un profesor, los factores asociados de forma independiente con el rechazo a largo plazo eran el sexo masculino, el bajo rendimiento en Educación física, la imagen sexual de uno mismo negativa y los problemas de interiorización. Puede observarse que estos factores eran estadísticamente significativos en el modelo de regresión logística aunque el número de alumnos que sufrían rechazo por parte de sus compañeros era bajo.

Los chicos demostraron ser más propensos a ser rechazados que las chicas. Ya, en estudios anteriores, se demostró cómo la agresión y el rechazo eran más constantes entre los chicos que entre las chicas (Huesman et al. 1984, Pukkinen \& Pitkänen 1993). Las razones para explicar estos datos no se conocen aunque una podría ser la diferencia existente entre las normas sociales para los chicos y para las chicas. Por ejemplo, defender a las víctimas es más aceptado entre las chicas que entre los chicos y quizás para una chica ésta sea una forma efectiva de mejorar su estatus a los ojos de sus compañeros (Salmivalli et al. 1996). Otra explicación podría estar en el hecho de que sentirse rechazado altera la autoestima de un chico antes que la de una chica. Nuestra conclusión se apoya en una teoría anterior, según la cual, la utilización constructiva de la agresión es una parte fundamental en el proceso de una identidad masculina saludable, facilitando el distanciamiento progresivo de los padres así como la integración de la sexualidad masculina (Blos 1982). La mayor sensibilidad de los chicos ante los efectos negativos de los factores que producen estrés también ha sido reseñado en otros estudios (Rolf \& Wirt 1984, Johnson 1988, De Rosier, Kupersmidt \& Petterson 1994).

Según la valoración de los profesores, los alumnos que habían sido rechazados tenían en el inicio del estudio más problemas de interiorización y de hiperactividad en clase que el resto. Estas observaciones sugieren que los problemas de los alumnos que han sido rechazados durante un largo periodo de tiempo deben ser intrapsíquicos. Los estudios sincrónicos (Interbitzen, Walters \& Bukowski 1997, Hecht, Indderbitzen \& Bukowski 1998) y los de seguimiento o diacrónicos (Roff \& Wirt 1984, Kupersmidt \& Coie 1990, Newcomb, Bukowski \& Pattee 1993, Bell-Dolan, Foster \& Christopher 1995) han mostrado que los alumnos que padecen el rechazo de otros son más ansiosos y tienen más actitudes negativas hacia 
los demás. Todos estos factores llevan a un aislamiento social con respecto a otros alumnos,pudiendo el rechazo fomentar problemas mentales.

Los alumnos que habían sido rechazados tenían una imagen negativa de sí mismos, tanto en el aspecto sexual como en el social. Esos resultados parecen reflejar el hecho de que estos adolescentes tienen dificultades para hacer frente a los desafíos que supone el desarrollo en la adolescencia, especialmente los cambios físicos de la pubertad, la madurez sexual y las relaciones con los compañeros. Ya que pudimos determinar el periodo de duración de la pubertad como un elemento de confusión, nuestros resultados sostienen la importancia de un desarrollo intrapsíquico que se ha llevado a cabo con éxito para lograr una adaptación social positiva durante la adolescencia.

Las dificultades de desarrollo pueden manifestarse especialmente durante las clases de educación física, cuando los alumnos tienen que utilizar no sólo su cuerpo sino también sus habilidades sociales, ya que las clases de esta asignatura no están tan orientadas hacia una sola tarea como otras clases. La suposición de que existe una relación entre el desarrollo psicológico y el significado que tienen las relaciones entre compañeros, se apoya en la observación de O’Koo (1997) y también en nuestros descubrimientos anteriores (Lakkanen et al. 1998), todo ello de acuerdo con el efecto que tiene la relación con los compañeros en la construcción de la imagen de sí mismo y en la adaptación psicológica al crecimiento físico y a la maduración sexual durante la adolescencia.

Los alumnos que tomaron parte en nuestro estudio representaban el $26 \%$ de la cohorte de edad de aquellos que asistían a clase en los centros de Educación Secundaria en las ciudades finlandesas de tipo medio. El número de alumnos rechazados era bajo, lo que aumenta la posibilidad de un error del tipo 2 (por ejemplo existían diferencias reales pero no se observaron). Por otro lado, el $92 \%$ de los alumnos que tomaron parte en el inicio del estudio, participaron en el seguimiento, y esto reduce el riesgo de parcialidad en la selección.

La fiabilidad de nuestros resultados aumenta gracias a la gran cantidad de información que proporcionaron diferentes informadores y al uso de métodos que habían sido probados y estructurados con anterioridad.

El rechazo a largo plazo puede perjudicar el desarrollo y la autoestima de los adolescentes (Roff \& Wirt 1984, Youniss \& Haynie 1992, Newcomb, Bukowski \& Pattee 1993). Tanto los niños como los adolescentes consideran que el rechazo a largo plazo supone para ellos un estrés tan serio como el de perder a un amigo (Johnson 1988). Con anterioridad, se había demostrado que las observaciones que llevan a cabo los profesores son valiosas y pueden ser cruciales para la detección temprana de problemas en sus alumnos (Ollendick et al. 1992, Olin, John \& Mednick 1995).

\section{Conclusión}

Se debe identificar a aquellos alumnos que corren el riesgo de ser rechazados y se han de tomar las medidas necesarias para prevenir su marginación. Según nuestros resultados, el grupo de riesgo está compuesto por adolescentes con problemas de interiorización y por aquellos cuyo rendimiento en Educación física es bajo. Los chicos corren mayor riesgo que las chicas. 


\section{Bibliografía}

AHO, S. The Social types in class (in Finnish). Liedon työrauhakokelluprojekti: raportti 12. Turku: Turun yliopiston kasvatustietelden julkaisusarja A 63, 1979.

BELL-DOLAN, D.J.,FOSTER, S.L.,CHRISTOPHER, J.S. Girls, peer relations and internalizing problems:Are socially neglected, rejected and withdrawn girls at risk? Journal of Clinical Child Psychology 24:463-473, 1995.

BLOS, P. On adolescence. London, The Free Press,pp. 52-156, 1962.

BLOS, P. 1982. The adolescent passage. New York,Int Univ Press, INC, pp. 141-170, 1982.

BOLTON, M., SMITH, P.K. Bully/victim problems in middle-school children: Stability, self-perceived competence, peer perceptions and peer acceptance. British Jour nal of Developmental Psychology 12: 315-29, 1994.

BOIVIN, M.,HYMEL, S., BUKOWSKI, W.M. The roles of social withrawal, peer rejection, and victimization by peers in predicting loneliness and depressed mood in childhood. Development and Psychopathology 7: 765-785,1995.

Classification of the socio-economic groups. Helsinki, Tilastokeskus, käsikirja 17, 1989.

COIE, J.D., DODGE, K.A., KUPERSMIDT, J.B. Peer group behavior and social status. In: Peer Rejection in Childhood. ASHER S.R., COIE J.D. (eds). New York, Cambridge University Press, pp. 17-59, 1990.

DEROSIER,M.E.,KUPERSMIDT, J.B., PATTERSON, C.J. Children's academic and behavioral adjustment as a function of the chronicity and proximity of peer rejection. Child Development 65: 1799-1813, 1994.

DOWNS, W.R., ROSE, S.R. The relationship of adolescent peer groups to the incidence of psychosocial problems. Adolescence 26: 473-492, 1991.

FOREHAND, R.,WIERSON, M., McCOMBS, THOMAS, A., ARMISTEAD, L., KEMPTON, T., NEIGHBORS, B. The role of family stressors and parent relationships on adolescent functioning. American Academy of Child and Adolescent Psychiatry 30: 316-322, 1991.

FRANZOI, S.L., DAVIS, M.H., VASQUEZ-SUSON, L.A. Two social worlds: social correlates and stability of adolescent status groups. Journal of Personality and Social Psychology 67: 462-473, 1994.

GIORDANO, P.C., CERNKOVICH, S.A., GROAT, H.T.,PUGH,M.D.,SWINFORD, S.P. The quality of adolescent friendships: long-term effects? Journal of Health and Social Behavior 39: 55-71, 1998.
GRESHAM, F.M., ELLIOT, S.N. Social Skills Rating System. Manual. American Guidance Service. Circle Pines, 1990.

HECHT, D.B., INDDERBITZEN, H.M., BUKOWSKI, A.L. The relationship between peer status and depressive symptoms in children and adolescents. Journal of Abnor mal Child Psychology 26: 153-160, 1998.

HUESMAN, R., ERON, L., LEFKOWITZ, M., WALDER, L. Stability of aggression over time and generations. Developmental Psychology 20: 1120-1134, 1984.

INDERBITZEN, H.M., WALTERS, K.S., BUKOWSKI,A.L. The role of social anxiety in adolescent peer relations: differences among sociometric status groups and rejected subgroups. Journal of Clinical Child Psychology 26: 338-348, 1997.

JOHNSON, J.H. Life events as stressors in childhood and adolescence. Newbury Park, CA: Sage, 1988.

KALTIALA-HEINO, R.K., RIMPELÄ, M., MARTTUNEN, M., RIMPELÄ, A., RANTANEN, P. Bullying, depression, and suicidal ideation in Finnish adolescents: school survey. British Medical Journal 319: 348-351,1999.

KANDEL, D.B., DAVIES, M. High school students who use crack and other drugs. Archives of General Psy chiatry 53: 71-80, 1996.

KUPERSMIDT, J.B., COIE, J.D. Preadolescent peer status, aggression, and school adjustment as predictors of externalizing problems in adolescence. Child Development 61: 1350-1362, 1990.

LAUKKANEN, E., HALONEN, P.,VIINAMAKI, H. Stability and internal consistency of the Offer Self-lmage Questionnaire. A study of Finnish adolescents. Journal of Youth and Adolescence 28: 71-77, 1999a.

LAUKKANEN, E., LEHTONEN, J., AMNELL, G., VIINAMAKI, H. Body image and psychopathology in adolescence:A controlled clinical study. Acta Psychiatrica Scandinavica 98: 47-53, 1998.

LAUKKANEN, E., PEIPONEN, S., HALONEN, P., AIVIO, A.,VIINAMÄKI, H. Discriminant validity of the Offer Self-lmage Questionnaire in Finnish 13-year-old adolescents. Nordic Journal of Psychiatry 53: 197-201, 1999b.

LEVINE, S.V. The anxieties of adolescents. Journal of Adolescent Health Care 2: 133-137, 1981.

NEWCOMB, A.F., BUKOWSKI, W.M., PATTEE, L. Children's peer relations: a meta-analytic review of popular, rejected, neglected, controversial and average sociometric status. Psychological Bulletin 113, 99-128, 1993. 
O'KOON, J. Attachment to parents and peers in late adolescence and their relationship with self-image. Adoles cence 32: 471-482, 1997.

OFFER, D., OSTROV, E.,HOWARD, K.J.,DOLAN, S. Offer Self-lmage Questionnaire, revised. Los Angeles, Western Psychological Services, 1992.

OLIN, S.S., JOHN, R., MEDNICK, S.A. Assessing the predictive value of teacher reports in a high risk sample for schizophrenia: a ROC analysis. Schizophrenia Research 16: 53-66, 1995.

OLLENDICK, T.H., WEIST, M.D., BORDEN, M.C., GREENE, R.W. Sociometric status and academic, behavioral, and psychological adjustment: a five-year longitudinal study. Journal of Consulting and Clinical Psychology 60: 80-87, 1992.

PETERSEN, A.C. Adolescent development. Annual Review Psychology 39: 538-607, 1988.

PULKKINEN, L., PITKÄNEN, T. Continuities in aggressive behavior from childhood to adulthood. Aggres sive Behavior 19: 249-263, 1993.

RESNICK, M.D., BEARMAN, P.S., BLUM, R.W., BAUMAN, K.E.,HARRIS, K.M.,JONES, J., TABOR, J., BEUHRING, T.,SIEVING, R.E.,SHEW, M.,IRELAND, M., BEARINGER, L.H., UDRY, J.R. Protecting adolescents from harm. Findings from the National Longitudinal Study on adolescent health. Journal of the American Medical Association 10, 278: 823-832, 1997.
ROFF, J.D., WIRT, R.D. Childhood social adjustment, adolescent status, and young adult mental health. Ameri can Journal of Orthopsychiatry 54: 595-602, 1984.

SALMIVALLI,C.,LAGERSPETZ,K.,BJORKQVIST, K.,OSTERMAN, K.,KAUKINEN, A. Bullying as a group process: Participant roles and their relations to social status within the group. Agressive Behavior 22: 1-15, 1996.

SHULMAN, S. Close relationships and coping behavior in adolescence. Journal of Adolescence 16:267-283,1993.

STENSMSEN, S. Rejected pupils in the school class. Pedagogisk Forskning 9: 125-145, 1965.

WENTZEL, K.R., CALDWELL, K. Friendships, peer acceptance, and group membership:Relations to academic achievement in middle school. Child Development 68: 1198-1209, 1997.

YOUNISS, J., HAYNIE, D. Friendship in adolescence. Journal of Development andBehavioral Pediatrics 13:5966, 1992.

Dirección para correspondencia:

Dr. Eila Laukkanen

Psiquiatra de Adultos y Adolescentes

Kuopio University Hospital

P.O. Box 1777,

FIN-70211 Kuopio

Fax +358-17-172986

FINLANDIA 